\title{
EFFECTS OF FORCING DATA RESOLUTION IN RIVER DISCHARGE SIMULATION
}

\author{
Roshan SHRESTHA ${ }^{1}$, Yasuto TACHIKAWA ${ }^{2}$ and Kaoru TAKARA ${ }^{3}$ \\ ${ }^{1}$ Graduate Student, Dept. of Civil Engineering, Kyoto University, (Gokasho, Uji, 611-0011, Japan) \\ ${ }^{2}$ Member of JSCE, D. Eng., Associate Professor, DPRI, Kyoto University, (Gokasho, Uji, 611-0011, Japan) \\ ${ }^{3}$ Member of JSCE, D. Eng, Professor, DPRI, Kyoto University, (Gokasho, Uji, 611-0011, Japan)
}

\begin{abstract}
Macro scale distributed hydrological models simulate river discharge with better accuracy but it depends upon the grid resolution of input data. Effects of different input resolutions are studied here. Three different grid resolution input data obtained from HUBEX-IOP EEWB data and GAME Re-analysis data are used to simulate river discharge and compared against the observed one. GAME Re-analysis 1.25-degree resolution data are found quite satisfactory in larger basins, while HUBEX-IOP EEWB 10-minute resolution data are better for small catchments. GAME Re-analysis 2.5-degree resolution data did not give good result. Simulated results by using spatially interpolated data are rather worse than using original data. The Huaihe River basin $\left(132350 \mathrm{~km}^{2}\right)$ is taken as the case of study.
\end{abstract}

\section{Key Words : GAME Reanalysis data, HUBEX-IOP EEWB data, macro scale hydrologic model}

\section{INTRODUCTION}

Hydrologists often practice discharge simulation not only to know the flow pattern but also to use the flow data information in several decision-making and strategy formulation processes. However, correct prediction of river discharge is still a challenging job.

Macro scale hydrological models are tools to predict the river discharge. However their results largely depend on the quality of input data and grid resolution. Garbage in garbage out (GIGO) applies here as well. Very high-resolution data may not be necessary especially on the verge of catchment size as it adds cost. Using minimum data to obtain better result is always highly prioritized. Suitability of grid resolution to a certain range of catchment size may be matter of discussion. In this study, effects of grid resolution of forcing data in river discharge simulation are examined.

Basin and sub-basins of Huaihe Kiver in China are taken as the case to study. Grid precipitation and actual evapo-transpiration data are referred from (1) HUBEX IOP EEWB data with 5-minute spatial and 1 hour temporal resolution, (2) GAME Reanalysis data (Version 1.1) with 1.25-degree spatial and 6 hour temporal resolution and (3) GAME Reanalysis data (Version 1.1) with 2.5-degree spatial and 6 hour temporal resolution for the period from May1, 1998 to August 31, 1998. The effects of data interpolation in distributed hydrologic simulation are also presented. A brief description of the model and findings are presented in this paper.

\section{MODEL DESCRIPTION}

A macro scale grid based distributed hydrological model $^{1,2), 3)}$ is used here. It simulates water movement following automated procedures like basin partitioning, sub-basin hydrological modeling and linking sub-basin models together to produce total run-off with the use of OHyMoS, Object-oriented Hydrological Modeling System ${ }^{4)}$. Main features of the model are as follows:

(a) This model is able to consider exact location and linkage of river segments even within a grid-cell on physical basis to give better flow routing, which has dominant effect on hydrograph.

(b) Dividing watershed basin into grid-cells according to a meso-scale atmospheric model, this model incorporates the atmospheric model output into hydrological model effectively. For each grid-cell, a runoff process and a flow routing process are modeled separately before constructing a total runoff simulation system.

In addition, this model considers the channel width in accordance to the catchment coverage. 


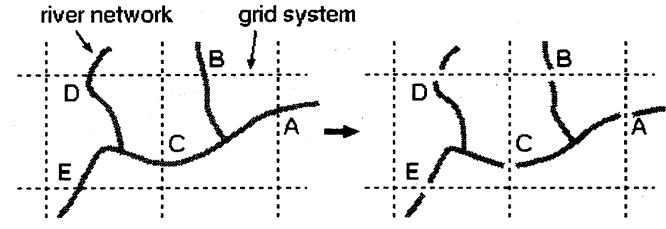

Fig.1 Division of river network

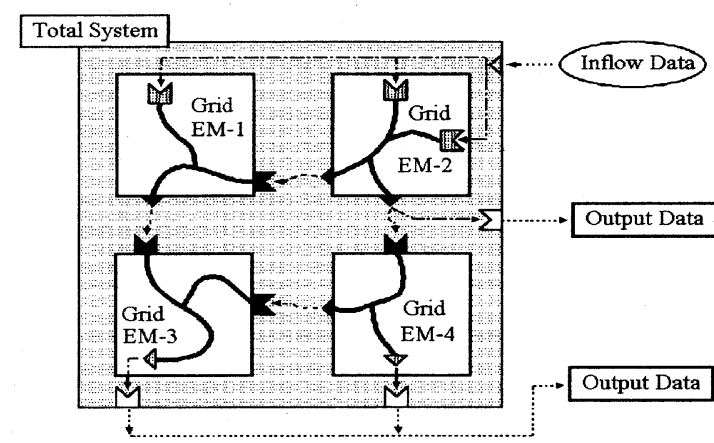

Fig.2 Schematic total hydrological simulation model

(1) River network arrangement

This model re-arranges the river network before simulation. 10-minute grid-cell frames divide existing river network into piecewise sub-networks and assigns new identity defining independent micro network within a cell. Figure 1 displays the process schematically. Existing network data (Fig. 1) intersects grid-cell frame at points A, B, C, D and E, which is re-arranged by dividing at $\mathrm{A}, \mathrm{B}, \mathrm{C}, \mathrm{D}$ and $\mathrm{E}$ to produce sub-networks within individual grid-cells.

\section{(2) Runoff Process Model (RPM)}

With the use of a simple hydrological model ${ }^{1)}$ based on Xinanjiang model ${ }^{5)}$ within a grid-cell to assess hydrological processes in view of spatial distribution of soil water capacity, the runoff appears only after soil water storage exceeds its capacity to hold.

\section{(3) Flow Route Model (FRM)}

A lumped stream kinematic wave model ${ }^{6)}$ computes discharge from outlets within each grid-cell. It assumes that the discharge varies linearly along each river segment at each time step instead of computing that for each computational cross-section.

\section{(4) Constructing total simulation process}

A total hydrological simulation model (Fig. 2) is constructed using functions of OhyMoS ${ }^{4)}$ to connect element models with "Data sending port (DSP)" and "Data receiving port (DRP)"

For each grid-cell, DRP feeds grid-mean input data (precipitation and evapo-transpiration) to runoff process model (RPM) and obtain grid-discharge. DSP feed that value into the flow route model (FRM). The FRM receives information

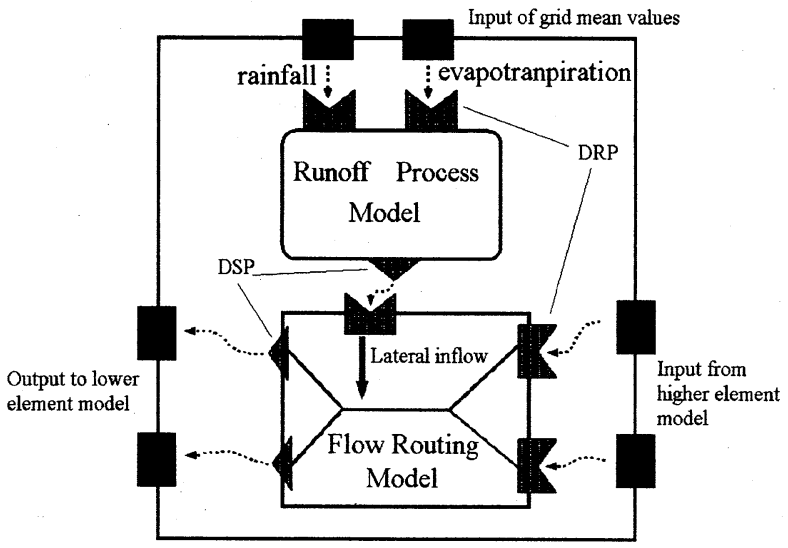

Fig.3 Structure of element model

Table 1 Catchment area of test basins.

\begin{tabular}{|c|l|c|}
\hline No. & Catchment Name & Area $\left(\mathrm{km}^{2}\right)$ \\
\hline \hline 1. & Suiping & 2093 \\
\hline 2. & Wangjiba & 29844 \\
\hline 3. & Bengbu & 132350 \\
\hline
\end{tabular}

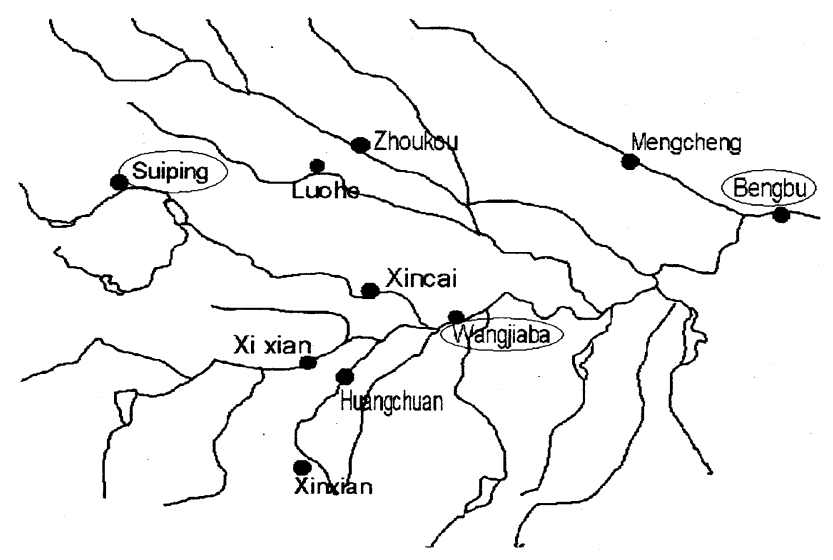

Fig.4 Location of basin confluences.

through its DRP from the RPM and adjacent upper element models (EMs) to compute discharge to the lower EMs (see Fig. 3). Before exchanging information between EMs, it automatically accumulates the calculated values and transfers that to lower EMs only on request.

Flow path of river network arrangement defines the upper and lower EMs. 'I'hey are linked in accordance with network connection relationship to transfer the computed outputs. Total runoff simulation model is thus a combination of EMs.

\section{BASIN AND SUB-BASINS}

The Huaihe River Basin and two sub-basins are taken for this study. Three points namely Suiping, Wangjiba and Bengbu (see Fig. 4) are adopted to compare the model output against the observed discharge. The largest catchment is Bengbu and smallest is Suiping (see Table 1). 


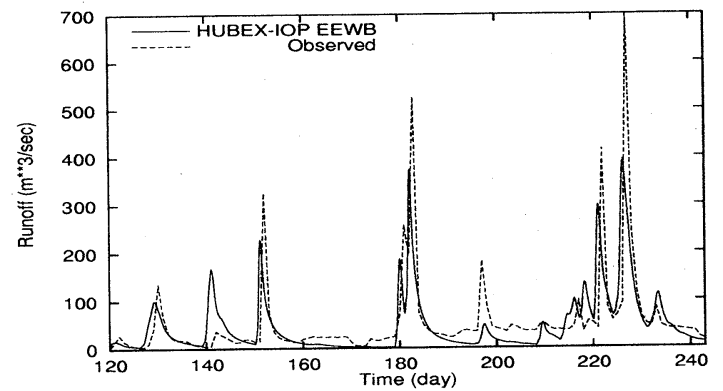

Fig.5 Simulation result for Suiping with HUBEX-IOP data.

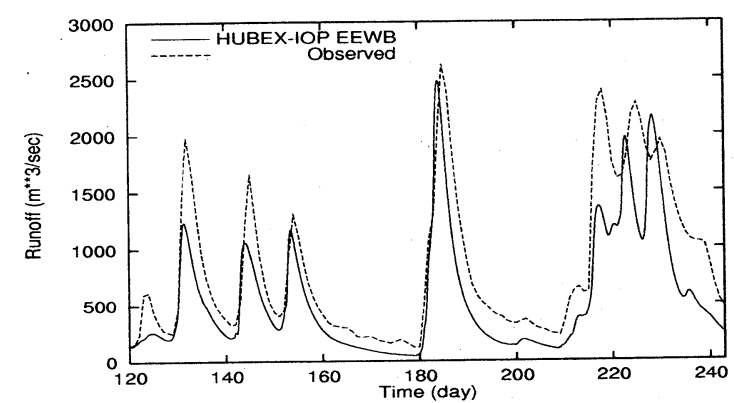

Fig.6 Simulation result for Wangjiba with HUBEX-IOP data.

\section{INPUT DATA DESCRIPTION}

The input hydrological data are precipitation and actual evapo-transpiration. Original data, after extraction, are fed into the model with resolution conversion. The simulation model accepts 10-minute spatial and 1-hour temporal resolution data. Therefore data resolution is converted as the simulation model's requirement. Following two kinds of data are used for discharge simulation and the results are compared.

\section{(1) HUBEX-IOP EEWB data ${ }^{7,8)}$}

A 5-minute grid resolution dataset, created for Estimation of Energy and Water Budget (EEWB) in the Huaihe River Basin, China is used in this study. It is based on Huaihe River Basin Experiment Intensive Observation Period (HUBEX-IOP).

Grid precipitation data are generated by interpolation of the ground observation points. This interpolation considers both distance and direction of each observation station. Hourly precipitation data are interpolated from 12 hour accumulated values. Grid evapo-transpiration data are generated from the observed surface meteorological data using SIBUC model ${ }^{8)}$.

\section{(2) GAME Re-analysis data ${ }^{9)}$}

Using 2-dimensional forecast data of GAME Reanalysis Product (Version 1.1) with 1.25 and 2.5-degree resolutions, the precipitation and actual evapo-transpiration data are extracted for all the grids lying in the basin.

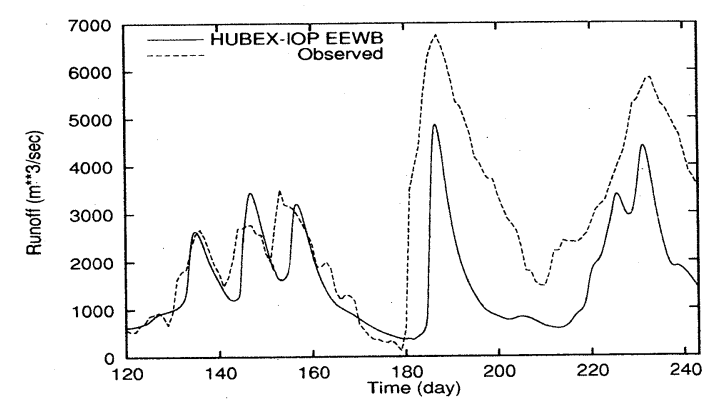

Fig.7 Simulation result for Bengbu with HUBEX-IOP data.

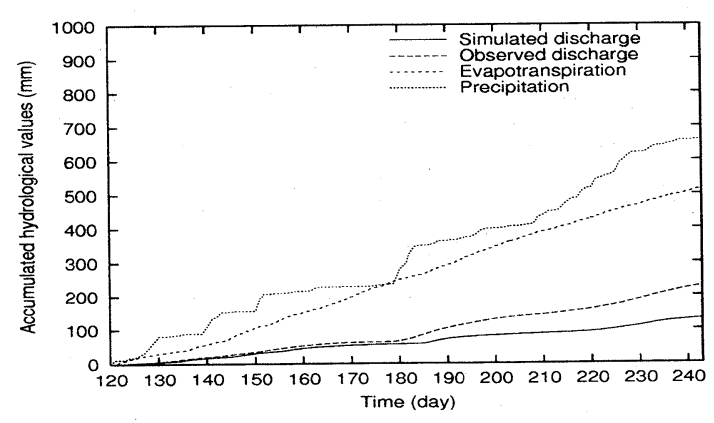

Fig.8 Water budget for Bengbu with HUBEX-IOP data.

These dataset is produced using JMA 4DDA system in a co-operative study of MRI/JMA, NPD/JMA and EORC/NASDA. This dataset has an advantage of including the GAME special observation off-line radio-sonde dataset ${ }^{10)}$. The temporal resolution of these data is 6-hour.

\section{DISCHARGE SIMULATION}

\section{(1) HUBEX-IOP EEWB data}

HUBEX-IOP EEWB data are converted to 10-minute spatial resolution by averaging the adjacent grid data before feeding into the hydrologic model. It gives quite satisfactory result while simulating the discharge with HUBEX-IOP EEWB data input in Suiping and Wangjiba catchment (see Figs. $5 \& 6$ ). However, the simulation remains good in Bengbu only for initial 60 days, and after then the runoff is predicted below observed values (see Fig. 7). The water budget (Fig. 8) clearly reflects this distinct difference between observed and simulated values of accumulated discharge, showing significant mismatch after $180^{\text {th }}$ days.

\section{(2) GAME Re-analysis data}

GAME Re-analysis data are re-arranged to fit according to the need of hydrological model's input requirement. While re-arranging input data, all 10 -minute grid cells, lying within 1.25-degree grid coverage, are assigned same value to maintain 1.25-degree resolution. The intersecting grid-cells are assigned average values. 


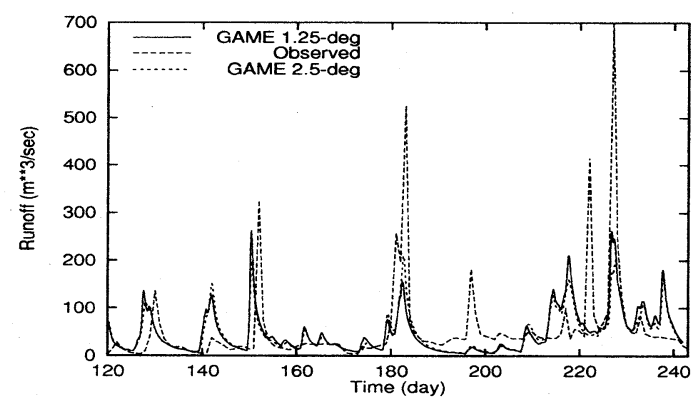

Fig.9 Simulation result for Suiping with GAME data.

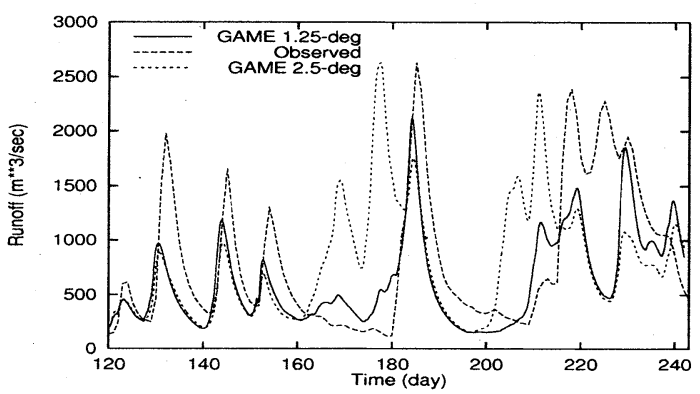

Fig.10 Simulation result for Wangjiba with GAME data.

The original 6-hour data are changed to 1-hour data assuming uniform distribution to maintain 6-hour interval discrete data. Thus the resolution of input data are maintained as 1.25-degree 6-hour data. Similar practice is applied in the case of 2.5-degree resolution as well.

\section{a) 1.25-degree resolution input}

The simulation results at Suiping using GAME 1.25-degree data input are not satisfactory, as it fails to attain the peak values several times (Fig. 9). Also, it is hard to follow the observed discharge at many places especially after $70^{\text {th }}$ days.

The simulation results at Wangjiba are nearly following the observed hydrograph but it fails at many places (Fig. 10). Distinct error is seen in attaining peak values and between $220^{\text {th }}$ to $230^{\text {th }}$ days, however trend is represented better.

It gives good result for Bengbu (see Fig. 11). The observed low flow in between $165^{\text {th }}$ and $180^{\text {th }}$ days are not represented properly, but the peak flow is simulated quite better than that with HUBEX-IOP EEWB data. The recession limb of hydrograph is still not simulated properly. Errors are present in further periods too but the trend is trying to follow the observed hydrograph. Looking over the accumulated discharge, it has given very good matching (Fig. 12) with observed one, and shows satisfactory agreement with the water budget.

\section{b) 2.5-degree resolution input}

This data did not give good simulation results in either basin (Fig. 9, 10, 11). In the water budget of Bengbu, accumulated discharge are nice in initial 50 days, and then there is great mismatch (Fig. 13).

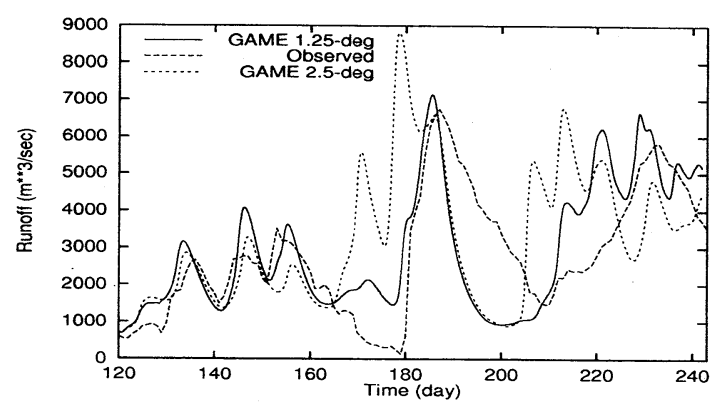

Fig.11 Simulation result for Bengbu with GAME data.

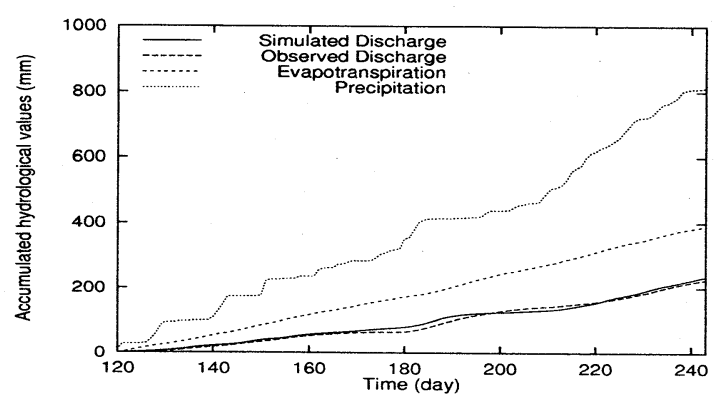

Fig.12 Water budget for Bengbu with GAME 1.25-deg data.

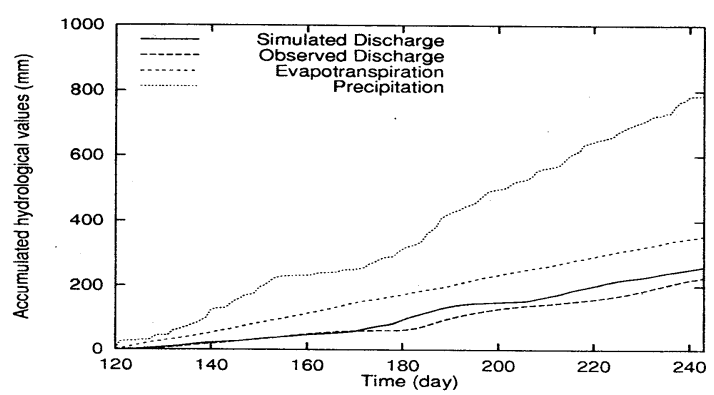

Fig.13 Water budget for Bengbu with GAME 2.5-deg data.

\section{(3) Why so much variation in result?}

The largest basin, Bengbu, touches seven 2.5-degree grid cells but only one lies completely within it. Diverse local hydrological and meteorological behaviors are impossiblc to bc reflected in coarse grid information. The river basin's diverse variability, which the present data does not reflect, may be influencing factor in flow routing process. Hence the mean value of all the locations within its coverage is believed as the major cause of failure to represent the true basin conditions. On the other hand, the results are quite satisfactory with 1.25 degree data, where, the same basin touches eighteen 1.25-degree grid-cells, among them ten grid-cells lie completely within it. This provides a base to accept that increased number of grid cell within catchment improves the result.

Looking over the smallest study basin Suiping, the simulated results are almost similar for 1.25-degree and 2.5 degree data - both not matching with observed one (Fig. 9). The grid area is higher than catchment area in both cases. So the simulation model assigns uniform spatial data, which does not represent the true site condition, producing much deviation in simulation result than the real one. 


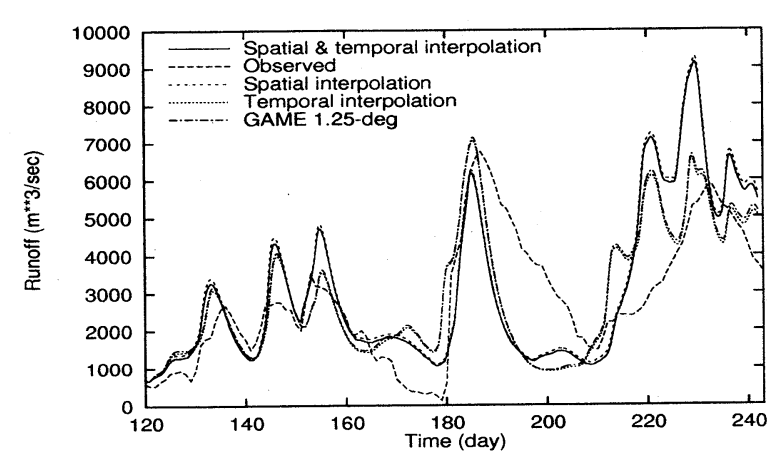

Fig.14 Simulation result with interpolated data for Bengbu

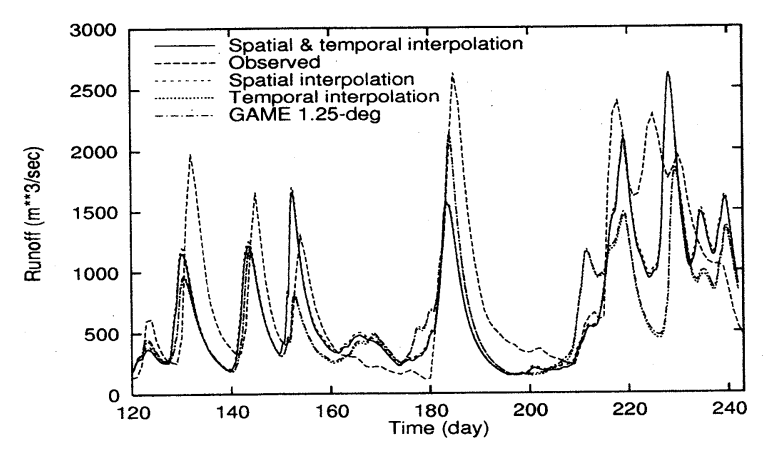

Fig.15 Simulation result with interpolated data for Wangjiba

\section{EFFECTS OF INTERPOLATION}

GAME 1.25-degree data and the Bengbu catchment are chosen for observing the effects of interpolation, as it is the best-simulated combination among the alternatives in previous section. Data are separately interpolated in space and time, and then fed into the model to observe the effects in discharge simulation.

\section{(1) Spatial interpolation data}

Assuming that the original grid data refers to the grid intersection points, the bilinear interpolation is applied before feeding the input data to the hydrological model. This experiment gave worse simulation result in Bengbu than the original data unlike the expectation (Fig. 14). The peak values did not match, although it is able to represent the trend. The error is quite distinctive after $220^{\text {th }}$ days of simulation in Bengbu.

In addition to that, the spatially interpolated data did not give better simulation result in Wangjiba and Suiping (Figs. 15, 16). Significant variations in simulation result are observed with respect to original data, as an effect of spatial interpolation.

\section{(2) Temporal interpolation}

Using linear interpolation, the original data is smoothened over 6-hour interval changing it into 1-hour time step discrete data. This data gave almost similar result as that of original data (Fig. 14, 15, 16). The output values are changed slightly but still they are not significant for all three basins.

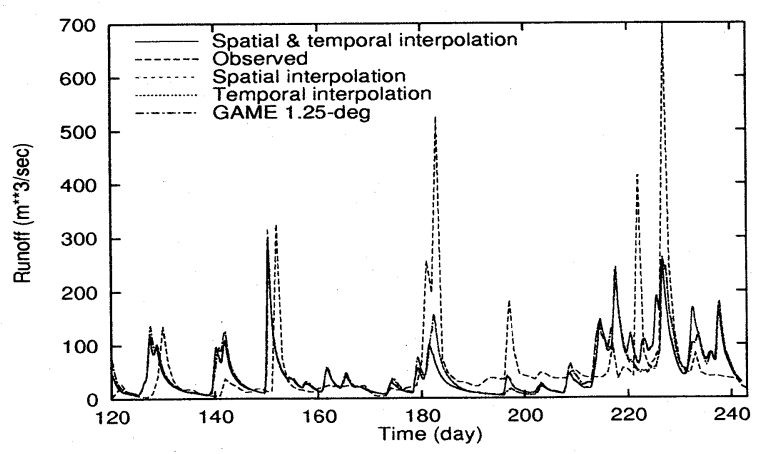

Fig.16 Simulation result with interpolated data for Suiping.

Table 2 Input-resolution-Catchment (IC) ratio.

\begin{tabular}{|l|c|c|c|}
\hline Resolution & Bengbu & Wangjiba & Suiping \\
\hline \hline $\begin{array}{l}\text { 2.5-degree } \\
\text { (GAME Re-analysis) }\end{array}$ & $1: 3.3$ & $1: 0.75$ & $1: 0.05$ \\
\hline $\begin{array}{l}\text { 1.25-degree } \\
\text { (GAME Re-analysis) }\end{array}$ & $1: 13.24$ & $1: 2.98$ & $1: 0.21$ \\
\hline $\begin{array}{l}\text { 10-minute } \\
\text { (HUBEX-IOP EEWB) }\end{array}$ & $1: 744.5$ & $1: 167.9$ & $1: 11.78$ \\
\hline
\end{tabular}

\section{(3) Spatial and Temporal interpolation}

Integrating both spatial interpolation and temporal interpolation a new data with 10-minute spatial and 1-hour temporal resolution data is created. It gives almost similar result as that of spatial interpolation only (Figs. 14, 15, 16). This means that spatial interpolation has dominant effect for discharge simulation.

\section{WHAT DOES IT SUGGEST?}

The obtained results clearly indicate that distributed hydrological models are very sensitive with resolution of forcing data. Of course, the accurate higher resolution data gives better solution. If we see over the ratio of input-resolution and catchment size (IC ratio), it indicates that there exists some ratio, which marginates the suitability of largest forcing data resolution to input in the distributed hydrological model with respect to catchment size. A summary of IC ratios is presented in Table 2.

While input-resolution-catchment (IC) ratio is 1:13, it gave good result at Bengbu. The results are found satisfactory with the IC ratio is $1: 168$ at Wangjiba and with the IC ratio 1:11 at Suiping. Simulation is not satisfactory with lower IC ratios. It may be too early to draw some specific conclusion, but still this study suggests that the results of distributed hydrologic models are better while IC ratio is more than that of $1: 10$. To set a general acceptable IC ratio or to investigate the relationship of IC ratio with other hydrological factors may be a good research topic in the coming days. 
The results of interpolated data display that the effect of spatial interpolation is dominant in distributed hydrologic simulation than the temporal interpolation. This means the spatial distribution effect is very sensitive. It is obvious to have less effect of temporal distribution in large basins like Bengbu and Wangjiba because of larger travel time, but it did not affect much in the simulation result of Suiping also. This phenomenon is present in the result with spatially and temporally interpolated data as well.

The higher grid resolution is not necessarily good in hydrological modeling. Basically, the high-resolution data generation using interpolation techniques from the coarse grid intervals may have poor performance than the original one for large catchments. Spatially interpolated input data to model flow at Bengbu has shown this (Fig. 14). The larger grid data includes the average values within the grid cell but linear or hilinear spatial interpolation does not reproduce the data that considers topographical variations, catchment boundaries and hydrological characteristics exactly. Therefore, in distributed hydrological modeling, it cannot simulate the hydrological behavior of zonal variation by applying such interpolation techniques.

In the study basin area, large numbers of dams are operating but this model does not consider the discharge manipulation due to dam operation. The obtained figures display some hints about the effect of dam operation. It does not distinctly indicate hydrograph attenuation even though recession parts are widened in observed hydrograph. To explain these behaviors more specifically, it needs further studies. If the discharge variations are due to effects of dam operation, then it will give a strong support to verify the suitability of GAME Reanalysis data in hydrological modeling processes.

\section{CONCLUSION}

Distributed hydrological models are very sensitive with resolution of forcing data input. HUBEX-IOP EEWB data gave good simulation results for Suiping and Wangiba basins at Huaihe region. GAME Re-analysis forecast 2.5-degree data is not suitable to model the river discharge. However, GAME Re-analysis 1.25-degree data gives good simulation result at Bengbu of Huaihe River Basin. It is able to show the pattern, but still unable to simulate peak flows. Better simulations are achieved while the IC ratio (Input-resolutionCatchment ratio) is more than 1:10. Spatial distribution of data has significant effect than the temporal distribution in distributed hydrologic modeling. Also, it revealed that increasing spatial grid resolution from coarse resolution data source using bilinear interpolation technique is not sure to improve the discharge simulation.

ACKNOWLEDGMENT: The contribution of providing HUBEX-IOP EEWB data by Dr. K. Tanaka, DPRI, Kyoto University and GAME Re-analysis data by Japan Meteorological Agency are highly acknowledged. The authors are also grateful to the Grants-in-Aid for Scientific Research, Encouragement of Young Scientists (A) 13750491 for their support.

\section{REFERENCES}

1) Tachikawa Y., Kawakami T., Ichikawa Y., Shiiba M. and Takara K.: Application of a macro grid based hydrological model to the Huaihe River Basin in China, Annual Inurnal. of Hydraulic Engineering, pp. 127 132, 2001.

2) Tachikawa Y., Takara K., Ichikawa Y. and Shiiba M.: Test Simulation of a macro scale distributed hydrological model for the Huaihe River Basin in China, Proc. of International GAME/HUBEX Workshop, Sapporo, pp. 139 146, 2000.

3) Tachikawa Y., Takara K., Ichikawa Y. and Shiiba M.: Development of a macro scale distributed hydrological model using an object-oriented hydrological modeling system. HydroInformaticsCD-ROM, 2000, Cedar Rapids, Iowa.

4) Ichikawa Y., Shiiba M., Tachikawa Y. and Takara K.: Object oriented hydrological modeling system, HydroInfromatics CD-ROM, 2000, Cedar Rapids, Iowa.

5) Zhao, R. J.: The Xinanjiang model applied in China, Journal of Hydrology, vol. 135 pp. 371 381, 1992.

6) Shiiba M., Ichikawa Y., Ikebuchi S., Tachikawa Y. and Takasao T.: Scale-up of a runoff model using GIS and an object-oriented hydrological modeling system. Proceedings of HydroGIS 96: Application of Geographic Information System in Hydrology and Water Resources Management, IAHS Publ. No. 235, pp. 63 70, 1996.

7) Kozan O., Tanaka K. and Ikebuchi S.: Estimation of Energy and Water Budget in the Huaihe River Basin, China, Game publication no. 28 pp. 32-35, 2001.

8) Tanaka, K.: Activities in Huaihe River Basin (HUBEX) study area, Asia-Pacific Network for Global Change Research, Report of 1997-1998, pp. 69-91, 1998.

9) ftp://hydro.iis.u-tokyo.ac.jp/GAME/GAIN/GAME-REANL/

10) Yatagai A., Miyazaki S., Sugita M., Tsukamoto O., Ohte N. and Toda M.: A comparative study of surface fluxes derived from four dimensional data assimilation products with AAN observations, Game publication no. 28, pp. 25-28, 2001.

(Received October 1, 2001) 